\title{
Contemporary therapeutics and new drug developments for treatment of Fabry disease: a narrative review
}

\author{
Daniel Oder^, Jonas Müntze^, Peter Nordbeck^ \\ Department of Internal Medicine I, Fabry Center for Interdisciplinary Therapy (FAZIT) and Comprehensive Heart Failure Center (CHFC), \\ University Hospital Würzburg, Würzburg, Germany \\ Contributions: (I) Conception and design: All authors; (II) Administrative support: None; (III) Provision of study materials or patients: None; (IV) \\ Collection and assembly of data: None; (V) Data analysis and interpretation: All authors; (VI) Manuscript writing: All authors; (VII) Final approval of \\ manuscript: All authors. \\ Correspondence to: Peter Nordbeck, MD. Department of Internal Medicine I - Cardiology, Fabry Center for Interdisciplinary Therapy (FAZiT), \\ University Hospital Würzburg, Oberdürrbacher Str. 6, 97080 Würzburg, Germany. Email: nordbeck_p@ukw.de.
}

\begin{abstract}
Fabry disease (OMIM 301500) is an X-linked (Xq22.1) lysosomal storage disorder leading to a progressive multisystem disease with high variability in both genotype and phenotype expression. The pathophysiological origin is found in an enzyme deficiency of the $\alpha$-galactosidase A (enzyme commission no. 3.2.1.22) leading to accumulation of globotriaosylceramides in all lysosome carrying tissue. Especially organ manifestations of the heart, kidneys and nervous system are of significant prognostic value and might complicate with Fabry-associated pain, young aged cryptogenic stroke, proteinuria, kidney failure, hypertrophic cardiomyopathy, heart failure, malign cardiac rhythm disturbances and eventually sudden cardiac death. Up to the introduction of the first enzyme replacement agent in 2001, patients faced the disease's natural course with no disease-specific therapies available. Today, two recombinant enzyme replacement agents $\left(\right.$ Fabrazyme $^{\circledR}$, Sanofi Genzyme, Cambridge, MA, USA; Replagal ${ }^{\circledR}$, Takeda Pharmaceutical, Tokio, Japan) and one oral chaperone therapy (Migalastat ${ }^{\circledR}$, Amicus Therapeutics, USA) are available and well-established in daily clinical practice. Substrate reduction therapy, second-generation enzyme replacement agents and different gene therapy approaches are currently undergoing preclinical and clinical trial phases and aim to improve therapeutic success and long-term outcome of patients with Fabry disease. This narrative review summarizes the currently available therapeutic options and future perspectives in Fabry disease.
\end{abstract}

Keywords: Fabry disease; enzyme replacement therapy; chaperone therapy; substrate reduction therapy; gene therapy

Submitted Aug 26, 2020. Accepted for publication Dec 09, 2020.

doi: $10.21037 / \mathrm{cdt}-20-743$

View this article at: http://dx.doi.org/10.21037/cdt-20-743

\section{Introduction}

Fabry disease (FD; OMIM 301500) is an X-linked (Xq22.1) lysosomal storage disorder taking origin in enzyme deficiency of the $\alpha$-galactosidase A (enzyme commission no. 3.2.1.22) (1). This results into accumulation of globotriaosylceramides
(Gb3) and the derivative globotriaosylsphingosine (lysoGb3) in all lysosome carrying tissue. Clinically, phenotype expression is prominently accompanied by cardiac, renal and neurological impairments including young-aged cryptogenic stroke, chronic kidney disease, and a variant of hypertrophic

^ ORCID: Daniel Oder 0000-0002-7762-1652; Jonas Müntze 0000-0001-7494-159X; Peter Nordbeck 0000-0002-2560-4068. 
cardiomyopathy complicating with ventricular tachycardia and sudden cardiac death $(1,2)$.

Until 2001, no specific therapy was available accounting for poor prognosis especially in classical disease variants with multisystem manifestations (3). The introduction of enzyme replacement therapy (ERT), first approved in 2001, led to a significant improvement of complication rates and early death. However, some patients deal with insufficient response to therapy or antibody-related adverse events. Unfortunately, all benefits of ERT might come along with degrading quality of life due to the need of intravenous application. Thus, the first oral Fabry-specific drug in form of the chaperone Migalastat (Galafold ${ }^{\circledast}$, Amicus Therapeutics, USA) marked a further step to improved treatment options in FD. Dynamic drug development has ever since continued including substrate reduction and novel second-generation ERT agents waiting in line. Finally, gene therapy is moving forwards and first preliminary data has been presented. This review summarizes to date long-term results on first-generation ERT, the current state-of-the-art therapeutic options and gives a glimpse into the nearby future on Fabry-specific drugs. We present the following article in accordance with the Narrative Review reporting checklist (available at http:// dx.doi.org/10.21037/cdt-20-743).

\section{Methods}

We conducted a comprehensive systematic literature review considering literature in English and German language of articles on treatment of FD published up until April 2020 available on PubMed (https://pubmed.ncbi.nlm.nih.gov). Key words: Fabry disease; enzyme replacement therapy; chaperone therapy; substrate reduction therapy; gene therapy;

\section{Current treatment options}

In 2020 there are two different Fabry-specific therapy approaches with three drug agents available outside of ongoing clinical trials. Long-term data on efficiency and complications of ERT are broadly available, whereas realworld data on the relatively new oral chaperone still remain restricted to relatively short time frames.

\section{Long-term results on first-generation ERT}

Available since 2001, ERT has been the first commercially available Fabry-specific drug therapy. Almost 20 years after its first approval in Europe, several studies reporting longterm data on clinical outcome of first generation ERT have been published aiming to provide clinical guidance on the optimal time of initiation and dosage (4).

Currently, two recombinant ERT agents are approved and aim to supplement the either insufficiently available or defectively produced physiologic human $\alpha$-galactosidase A. While agalsidase alfa (Replagal $^{\circledR}$, Takeda Pharmaceutical, Tokio, Japan) is produced using human fibroblast lineages and administered in a dose of $0.2 \mathrm{mg} / \mathrm{kg}$ bodyweight, agalsidase beta (Fabrazyme ${ }^{\circledR}$, Sanofi Genzyme, Cambridge, MA, USA) is usually administered in a dosage of $1.0 \mathrm{mg} / \mathrm{kg}$ and is produced using Chinese hamster ovary cells. Both agents are advised to be given intravenously every other week and have been shown save in various randomized controlled trials (3,5-10).

Eng et al. reported that ERT leads to Gb3-deposition clearance in glomerular endothelial cells, which corroborated to clinical data indicating a significant reduction in occurrence of Fabry-associated events in patients undergoing ERT (11). Hughes and colleagues reported that initiation of agalsidase alfa led to a significant increase of the glomerular filtration rate indicating good therapeutic response of renal organ involvement (7). Other publications reported a slowed but progressive decline in glomerular filtration rate following ERT initiation (12-18). While the occurrence of albuminuria has effectively been prevented in patients negative for proteinuria at point of ERT-initiation, it was reported to persist in those with pre-existing albuminuria (19). Thus, therapeutic success seems to be patient- and case-depended varying from either improvement, stabilization, or further decline of kidney function. Similar results were reported for cardiac organ involvement with left-ventricular hypertrophy declining, stabilizing or proceeding on a case-dependent level $(16,20)$. Several studies indicate that ERT shows its best therapeutic potential in preventing disease progression and development of clinically relevant end-stage complications if started at early stages of disease where no irreversible organ damage has yet been set, while late therapy initiation might result in a diminished therapy efficacy (21-24). In this regard, Germain et al. reported that patients with no evident chronic kidney disease benefited more from agalsidase alfa therapy than patients presenting manifest kidney injury at point of ERT initiation (21). Similar results were published for cardiac organ involvement, were therapeutic effect was lower in patients presenting myocardial fibrosis at point of initiation (24). Furthermore, a multi-center study 
comparing lyso-Gb3 decrease after ERT initiation in men with classical FD who started ERT before versus after age 25 years, showed that early therapy initiation resulted into better biochemical response (23). As plasma lysoGb3 has been shown correlating with clinically feasible severity of FD, it seems to be a reliable biomarker besides of Gb3 deposition clearance on a histological level and thus presumably a valid indicator of therapy success, respectively failure $(25,26)$. While a trend towards a reduction of plasma Gb3 and lyso-Gb3 levels has been reported for both currently available ERT agents (6,21,27-36), some data have indicated a higher potential on a significant reduction observed in patients treated with agalsidase beta compared to agalsidase alfa therapy $(27,28,30)$. A potential positive impact of dose increase or regime change into weekly infusions was neglected by Schiffmann et al., who reported no significant impact on plasma Gb3 levels following intensification of therapy intervals to $0.2 \mathrm{mg} / \mathrm{kg}$ or even $0.4 \mathrm{mg} / \mathrm{kg}$ agalsidase alfa once per week $(37,38)$.

Beginning in June 2009, viral contamination of agalsidase beta production facilities resulted into a worldwide drug shortage implying 'drug holiday', dose reduction, or product switch in most patients with FD who had previously received agalsidase beta therapy $(39,40)$. Many of those patients who had previously received the regular dose of $1.0 \mathrm{mg} / \mathrm{kg}$ every other week now underwent dose reduction to $0.3-0.5 \mathrm{mg} / \mathrm{kg}$ agalsidase beta, or were switched to agalsidase alfa (40). Especially the latter was controversial, as clinical experience on ERT regime changing was very limited by then $(17,41,42)$. While some studies indicate kidney function deterioration following therapy alterations in patients who had previously been stable on ERT $(40,43,44)$, others discuss a neutral or clinically nonrelevant impact of dose change, respectively therapy switch in patients with FD. Even though short-term observation by Smid et al. did not reveal an increase in adverse events after dose reduction to $0.5 \mathrm{mg} / \mathrm{kg}$, an increase of lyso-Gb3 levels still indicated a rising disease activity (17). However, the latter has not been confirmed by other studies $(29,45,46)$. In 2017, Pisani et al. performed a meta-analysis on seven studies focusing on the effects of therapy switch and in fact did not observe any significant differences in renal function during follow-up (47). In part contradictory results were observed in regard of cardiac organ manifestations and function deterioration, which however overall also remained stable with no clinically relevant disease progression being triggered by therapy alterations (45-47).

In conclusion, individual response, therapy success and disease-progression might significantly vary on a case-tocase basis with personal risk factors, such as genotype, age and gender, time of ERT-initiation, the patients' personal disease activity, and phenotype expression potentially serving as disease-modifying factors indirectly influencing therapeutic success $(3,22)$. Finally, positive effects of firstgeneration ERT come along with the burden of biweekly infusions and potentially severe directly infusion-related side effects (48). Anaphylactic or anti-drug antibody mediated reactions may indeed result into clinical complications in rare cases $(3,49-54)$.

\section{Chaperone therapy}

Limitations of ERT, such as its inability to cross the bloodbrain-barrier, and the burden of intravenous application has emphasized the need of new, preferably orally available Fabry-specific drugs. This demand was pleased in 2016 with the introduction of the first-in-class chaperone agent Migalastat (Galafold ${ }^{\circledR}$, Amicus Therapeutics, USA). Chaperones are small molecules binding and stabilizing the modified alpha-galactosidase A in amenable mutational variants of FD. Hereby, it facilitates lysosomal trafficking and increases lysosomal enzyme activity subsequently enhancing enzymatic degradation of Gb3 into excretable form of Gb2 $(55,56)$. In order to evaluate whether a pathogenic mutation is amenable to chaperone therapy, a Migalastat-specific assay measuring Migalastat-induced changes in human embryonic kidney (HEK) cells are transfected with DNA plasmids containing pathogenic $G L A$ variants (56). The criteria for amenability were defined as an increase of enzyme activity by at least 1.2 -fold above the baseline value, with an absolute increase of at least $3 \%$ compared to wild type enzyme activity (56). After a $G L A$ variant has been tested once, all results are available in a public database (www.galafoldamenabilitytable.com), allowing physicians a simple and efficient planning of therapy.

In the phase III licensing trial, Germain et al. reported that among the randomly assigned patients with amenable mutations, left ventricular hypertrophy decreased significantly within the interventional arm. Furthermore, unspecific but Fabry-associated symptoms such as diarrhea, reflux, and indigestion decreased. The slight decrease in glomerular filtration rate can be argued to be within physiological ranges, however, indicating no strong benefit regarding renal organ involvement of respective patients under observation (57). 
Hughes et al. compared ERT with chaperone therapy in the phase III ATTRACT study. 57 Fabry patients (44\% male) on stable ERT were randomized to switch to Migalastat for 18 months or stay on ERT. A significant decrease of left ventricular mass index (measured via echocardiography) was reported for the Migalastat group $(n=34)$. Migalastat and ERT had identical effects on renal function and lyso-Gb3 levels (58).

In the extension of the phase III FACETS trial, Germain et al. reported clinical benefit of Migalastat especially for male Fabry patients with classic phenotype. After 24 months, activity of alpha-galactosidase A increased, renal function was stabilized and left ventricular mass index was reduced in a subgroup of the FACETS trial (59). However, the examined group of patients was small $(n=14)$ and included only male patients with classic phenotype compared with a subgroup of nearly exclusively female patients $(n=36)$. Therefore, results should be interpreted carefully.

Even though the data published within the described randomized trials indicated a strong benefit especially regarding cardiac organ involvement, many aspects of the heterogeneous disorder FD remain unaddressed. Real world data from patients treated with the commercially available drug have shown that there might be specific positive effects in the myocardium exceeding positive ERT effects, such as reverse remodeling with a decrease in myocardial late enhancement in cardiac magnetic resonance imaging after initiation of treatment (60). However, the question which patients might benefit most from chaperonespecifically in direct comparison to ERT-is still mostly unanswered. Importantly, it has been shown that also among the amenable patients there can be vast differences in biochemical response to therapy, ranging from normalization of enzyme activity to only marginal changes in vivo $(60,61)$. In a monocenter real world collective, median enzyme activity increased rapidly from $29 \%$ to $44 \%$ of the normal wild-type activity ( $\mathrm{P}=0.01)$. Plasma lyso-Gb3 levels at one year were stable with a tendency for reduction in both females $(\mathrm{P}=0.35)$ and males $(\mathrm{P}=0.20)$, while a reduction of GFR over the first year of treatment was seen [creatinine: 0.94 (IQR 0.81-1.09) vs. 1.0 (IQR 0.77-1.18); $\mathrm{P}=0.021]$ (61). Interestingly, at one year, enzyme increase in patients correlated with myocardial mass reduction $(\mathrm{r}=-0.546 ; \mathrm{P}=0.044)$, but not with renal function $(\mathrm{r}=-0.086$; $\mathrm{P}=0.770)(61,62)$.

To better evaluate the safety along with cardiovascular, renal and patients-reported outcomes and disease biomarkers under real world conditions, a prospective multicenter observational study was initiated as early as 2017 (63). The results from 59 (28 females) patients with amenable mutations following twelve months of chaperone therapy strengthen the preliminary results from clinical trials and single center experience regarding therapeutic effectiveness, especially in regard of cardiac organ involvement. Oral Migalastat therapy was generally safe with no severe adverse events occurring within the 12-month observational period. In detail, female as well as male patients both showed a significant reduction of left ventricular mass index, which had been predefined as primary endpoint (63). The glomerular filtration rate slightly decreased over the first year of therapy in concordance to the licensing trial (57).

Of note, the irregular intake every other day leads to different days of therapy every week. Therefore, an estimation of adherence control in patients with Migalastat is required implicitly. The ongoing MALTA study (NCT03683966) investigates therapy adherence of Fabry patients receiving chaperone therapy. Further long term data are to be obtained in order to finally evaluate the definitive value, benefits, and potentially also risks of Migalastat therapy as compared to ERT, the therapeutic approach with longest experience so far.

\section{Adjunct therapy}

Besides the disease-specific therapeutics listed above, a broad spectrum of non-specific adjunct therapies are applied regularly in patients with FD (Table 1). These not only include non-FD-specific drugs, but also nonpharmacological therapeutic interventions such as renal replacement or cardiac device therapy.

Due to their reno- and vasculoprotective effects, many FD patients require angiotensin converting enzyme inhibitors (ACEi), respectively angiotensin receptor II blockage (ARBs) in case of hypertension or proteinuria and still compensated kidney function (64). Warnock and colleagues showed that the additional application of ACEi/ARBs resulted into a significant antiproteinuric effect in patients with severe Fabry nephropathy who were undergoing enzyme replacement therapy (65). However, as these agents do not address the underlying pathophysiologic mechanisms including Gb3 accumulation in podocytes, the long-term positive effect of ACEi/ARBs remains speculative and most likely largely dependent on the success of the Fabry-specific therapy (66). In end-stage Fabry nephropathy, renal replacement therapy including 


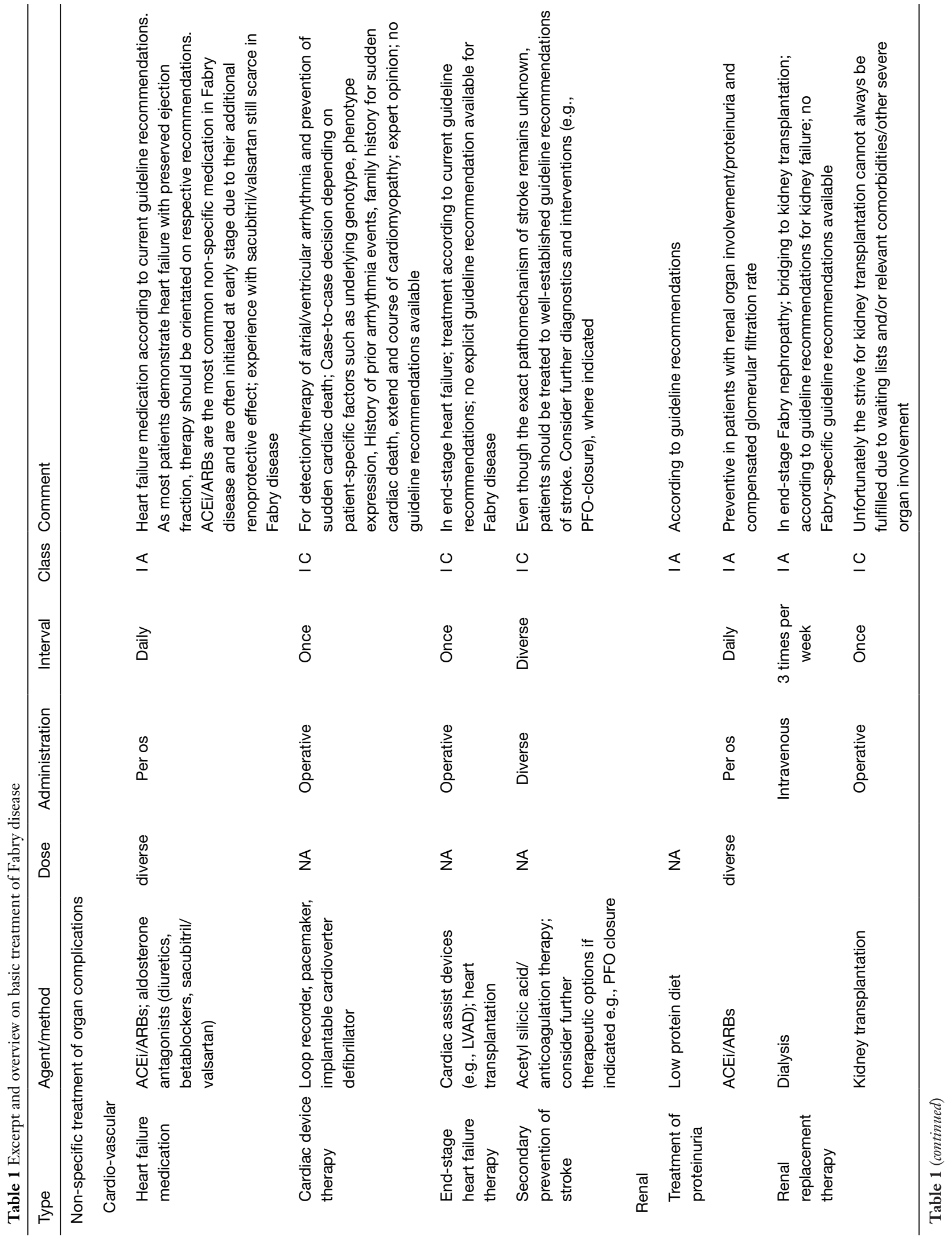




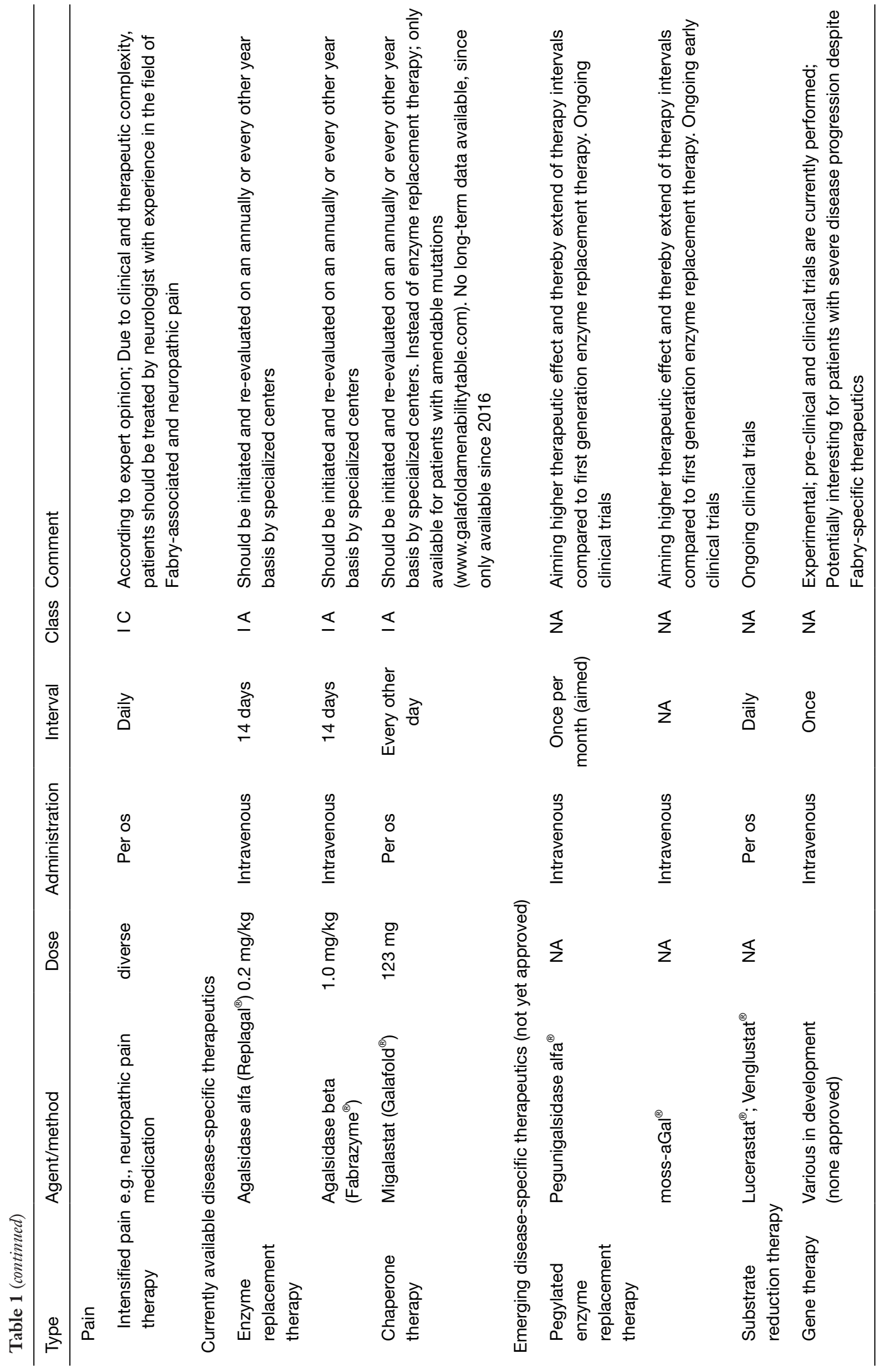


hemodialysis and kidney transplantation are well-established treatment options with results comparable to those in other diseases $(1,67,68)$.

Cardiac organ involvement and its clinical complications remain the main cause of premature death in $\mathrm{FD}$, highlighting importance of cardiac interventions. Fabry cardiomyopathy is a common cause of heart failure typically starting in the 3rd or 4th decade in life. Systolic function is usually preserved until late stages where left ventricular ejection fraction drops due to progressive scaring. The whole spectrum of heart failure medication might be applied taking into account clinical stage (69). Of note, betablockers are often not well tolerated by the patients, probably due to neurohumoral dysregulation. While aldosterone antagonists are well established, experience and long-term effects of novel therapeutics such as sacubitril/ valsartan are still limited in FD. Rhythm disorders are common, often resulting in the indication for pacemaker implantation. Despite the positive effects of Fabry-specific therapeutics, patients with advanced Fabry cardiomyopathy also still face a relevant burden of malign ventricular arrhythmia and sudden cardiac death. With only few published articles available, beneficial effects of implantable cardioverter defibrillator therapy remains inconclusive with no guidelines been established to date (70-73). This is of special relevance in those patients demonstrating with the "cardiac variants" where organ manifestations are almost completely limited to the heart. These patients often face a significant delay in identifying FD as underlying pathology, which can even worsen the risk of cardiac complications due to inadequate treatment (74). Clinical experience indicates that irreparable damage is often set at the point of FD diagnosis. Thus, the decision towards a recommendation of implantable cardioverter defibrillator therapy should be evaluated and discussed as a case-to-case decision especially in patients demonstrating with high-risk profile such as advanced Fabry cardiomyopathy with extensive replacement fibrosis, end-stage wall-thinning and/or prior ventricular tachycardia.

Clinical studies have also shown that FD is a relevant differential diagnosis in young-aged patients with cryptogenic stroke (75). In case of cerebrovascular events, diagnostic and therapeutic decisions should be performed according to current guideline recommendations for stroke (76). As treatment of Fabry-associated pain is complex, these patients should be referred to specialized neurologists for adequate pain therapy (77).

\section{New drug developments}

\section{Second-generation ERT}

While both first-generation lysosomal enzyme replacement agents are produced using mammalian cell lines, new "second-generation" ERT agents aim to overcome disadvantages of mammalian cell line production, including higher production costs and the risk of contamination by mammalian pathogens, and to extend therapeutic effects (78). As tissue uptake of intravenously applied enzyme replacement agents is usually performed though either the mannose or the mannose 6-phosphate receptor, it is assumed that these receptors might play a key role responsible for therapeutic efficiency of ERT in lysosomal storage disorders (78). Recent approaches aiming to establish lysosomal enzyme production in plant-derived cell lines have been reported before (79-81). For FD, two plant-derived ERT agents (Pegunigalsidase alf ${ }^{\circledR}$, Protalix Biotherapeutics, Israel; and moss-aGal ${ }^{\circledR}$, Greenovation biopharmaceuticals, Germany) are currently tested in clinical trials.

Pegunigalsidase alfa intends to not only increase plasma half-life of infused recombinant enzyme, but also to further improve long-term therapeutic tolerance by reducing the formation of anti-drug immunogenicity through pegylation of the enzyme (82). Murine models underlined the aimed therapeutic effect by prevention of Gb3 accumulation in both cardiac and renal tissues (83). Furthermore, clinical phase I/II trials also reported a reduction of Gb3 accumulation in human glomerular biopsy tissue acquired by kidney biopsies (82). First preliminary data of the phase III BRIDGE trial (NCT03018730) report that therapy switch from agalsidase alfa to Pegunigalsidase alfa was save, well-tolerated and resulted into stabilization, or at least slower progression of kidney failure (eGFR slope improvement from -5.1 to $0.23 \mathrm{~mL} / \mathrm{min} / 1.73 \mathrm{~m}^{2} /$ year in both male and female) (84). Further results of currently ongoing clinical trials (NCT02795676; NCT03018730, NCT03180840) evaluating Pegunigalsidase alfa treatment are expected to be published soon.

Different to the currently available mammalian cell based ERT agents, moss-aGal does not rely on mannose6-phosphate receptor mediated endocytosis but targets the mannose receptor for tissue uptake (85). Shen et al. evaluated the effectiveness of a non-phosphorylated $\alpha$ galactosidase A produced from moss (thus referred as "mossaGal") in an in-vitro and in-vivo mouse model of FD (78). Their key findings were that (I) endocytosis of moss-aGal 
was mannose receptor-mediated and dependent, (II) mossaGal was more preferentially targeted to renal cells than agalsidase alfa, (III) a single injection of moss-aGal resulted into a comparable substrate clearance in both cardiac and renal tissue as perceived in agalsidase alfa (78). Hennermann et al. conducted a phase I clinical trial evaluating moss-aGal therapy in six patients receiving a single dose of $0.2 \mathrm{mg} / \mathrm{kg}$ moss-aGal (86). In all patients, single dose moss-aGal application was well tolerated and showed a reduction of both plasma lyso-Gb3 levels (-3.8\%) and urinary Gb3 levels (up to $-60 \%$ ) after 28 days (86). Currently phase II/III trials further investigating this promising novel ERT-agent are in preparation.

\section{Substrate reduction therapy}

In contrast to currently available medication, substrate reduction therapy (SRT) neither aims to replace the insufficiently available or dysfunctional enzyme, nor to improve its activity by refolding. Instead, it blocks the emergence of Gb3 overload as well as its accumulation at an earlier step, namely at its point of production. Importantly, its therapeutic effects might also result into adverse events, as too intense abrogation of enzymatic reactions might result into homeostatic imbalance as discussed before (87). However, from the current point of view the unique selling point could become the ability of passing the blood-brain barrier, subsequently preventing cerebrovascular events, which to date remains an unmatched goal in Fabry-specific treatment (88). Two different SRT molecules (Venglustat ${ }^{\circledR}$, Sanofi Genzyme, Massachusetts, USA; and Lucerastat $^{\circledR}$, Idorsia Pharmaceuticals, Switzerland) have been developed for disease-specific treatment of FD and are currently evaluated in both pre-clinical and clinical trials (89-91). While Venglustat is still at an early stage of approval and only few data has been published so far. Preliminary data suggest a slow but gradual clearance of Gb3 from superficial skin capillary endothelium and a gradual decrease of plasma lyso-Gb3 in most therapy-naive patients (92). Lucerastat is currently undergoing clinical evaluation in the randomized multi-center double-blind clinical phase III MODIFY-trial (NCT03425539) (93). Promising initial results from phase I/II clinical trials show that therapy with lucerastat has been save with no clinically relevant safety abnormalities observed over a 12 -week long oral application (93). Furthermore, a significant decreased of plasma glycosphingolipids, glucosylceramide, lactosylceramide, and globotriaosylceramide compared to baseline values were observed rising hope for future application (93).

Both novel SRT agents are promising potential oral therapeutics for the nearby future with no limitation regarding specific mutations as seen in chaperone therapy.

\section{Gene therapy}

The strive for a curative therapy of FD intensified the efforts of enabling gene therapy over the last years. Different viral vectors have been tested and first FD patients already treated within early clinical phase I/II trials (NCT02800070; NCT03454893) (94). Current approaches comprise re-administration of lentiviruses-transfected haematopoetic stem cells (NCT02800070; NCT03454893), adeno-associated viral gene therapy $\left(F L T 190^{\circledR}\right.$, Freeline therapeutics, UK; and $S T-920^{\circledR}$, Sangamo Therapeutics) and micro-RNA based therapy (Moderna Inc; Translate Bio) (95-100). The first reports available so far have shown that the concept in general is valid, leading to a prompt substantial rise of alpha-galactosidase A levels in the first patients after treatment. However, it currently remains largely unclear whether these initial effects will be longlasting, or repetitive gene therapy will be needed. Future results of further clinical trials will further evaluate benefits but also risks, such as the development of neutralizing antibodies and immunologic reactions.

\section{Summary}

Treatment of FD in an exceptionally dynamic field with various new therapy approaches either already tested in clinical trials or arising in the near future. While ERT has proven its positive effects over many years, the availability of the first oral drug has led to a significant improvement at least of quality of life in many patients with FD already. However, due to amenability limitations, this approach is not an option for all patients with FD, currently leaving ERT essential as the only specific therapeutic option in most patients. Based on long-term experience and observational data from more than 20 years, early therapy initiation-before irreversible organ damage has occurred-is critically important, irrespective of the type of therapy chosen. Due to the rarity of FD, heterogeneity of the disease, and high therapy costs, therapeutic options should be thoroughly examined and re-evaluated annually thereafter in a specialized Fabry center, carefully taking the 
various factors such as individual risk, efficacy of therapy, and quality of life into account. With novel therapy options such as oral drugs but also gene therapy just arriving, this aspect becomes even more relevant in the future.

\section{Acknowledgments}

Funding: This work was supported by the Federal Ministry of Education and Research of the Federal Republic of Germany (BMBF 01EO1504, MO.2).

\section{Footnote}

Provenance and Peer Review: This article was commissioned by the Guest Editors (Yskert von Kodolitsch, Harald Kaemmerer, Koichiro Niwa) for the series "Current Management Aspects in Adult Congenital Heart Disease (ACHD): Part III" published in Cardiovascular Diagnosis and Therapy. The article has undergone external peer review.

Reporting Checklist: The authors have completed the NARRATIVE REVIEW reporting checklist. Available at http://dx.doi.org/10.21037/cdt-20-743

Peer Review File: Available at http://dx.doi.org/10.21037/ cdt-20-743

Conflicts of Interest: All authors have completed the ICMJE uniform disclosure form (available at http:// dx.doi.org/10.21037/cdt-20-743). The series "Current Management Aspects in Adult Congenital Heart Disease (ACHD): Part III" was commissioned by the editorial office without any funding or sponsorship. Dr. Oder reports personal fees from Sanofi, personal fees from Takeda, during the conduct of the study; personal fees from Sanofi, personal fees from Takeda, outside the submitted work. Dr. Müntze reports personal fees from Amicus, personal fees from Idorsia, grants and personal fees from Sanofi, personal fees from Takeda, during the conduct of the study; personal fees from Amicus, personal fees from Idorsia, grants and personal fees from Sanofi, personal fees from Takeda, outside the submitted work. Dr. Nordbeck reports grants and personal fees from Amicus, grants and personal fees from Idorsia, grants and personal fees from Sanofi, grants and personal fees from Takeda, during the conduct of the study; grants and personal fees from Amicus, grants and personal fees from Idorsia, grants and personal fees from Sanofi, grants and personal fees from Takeda, outside the submitted work. The authors have no other conflicts of interest to declare.

Ethical Statement: The authors are accountable for all aspects of the work in ensuring that questions related to the accuracy or integrity of any part of the work are appropriately investigated and resolved.

Open Access Statement: This is an Open Access article distributed in accordance with the Creative Commons Attribution-NonCommercial-NoDerivs 4.0 International License (CC BY-NC-ND 4.0), which permits the noncommercial replication and distribution of the article with the strict proviso that no changes or edits are made and the original work is properly cited (including links to both the formal publication through the relevant DOI and the license). See: https://creativecommons.org/licenses/by-nc-nd/4.0/.

\section{References}

1. Germain DP. Fabry disease. Orphanet J Rare Dis 2010;5:30.

2. Askari H, Kaneski CR, Semino-Mora C, et al. Cellular and tissue localization of globotriaosylceramide in Fabry disease. Virchows Arch 2007;451:823-34.

3. Oder D, Nordbeck P, Wanner C. Long Term Treatment with Enzyme Replacement Therapy in Patients with Fabry Disease. Nephron 2016;134:30-6.

4. Desnick RJ, Schuchman EH. Enzyme replacement therapy for lysosomal diseases: lessons from 20 years of experience and remaining challenges. Annu Rev Genomics Hum Genet 2012;13:307-35.

5. Banikazemi M, Bultas J, Waldek S, et al. Agalsidase-beta therapy for advanced Fabry disease: a randomized trial. Ann Intern Med 2007;146:77-86.

6. Eng CM, Guffon N, Wilcox WR, et al. Safety and efficacy of recombinant human alpha-galactosidase A replacement therapy in Fabry's disease. N Engl J Med 2001;345:9-16.

7. Hughes DA, Elliott PM, Shah J, et al. Effects of enzyme replacement therapy on the cardiomyopathy of Anderson-Fabry disease: a randomised, double-blind, placebo-controlled clinical trial of agalsidase alfa. Heart 2008;94:153-8.

8. Mehta A, Beck M, Elliott P, et al. Enzyme replacement therapy with agalsidase alfa in patients with Fabry's disease: an analysis of registry data. Lancet 2009;374:1986-96.

9. Schiffmann R, Kopp JB, Austin HA 3rd, et al. Enzyme replacement therapy in Fabry disease: a randomized 
controlled trial. JAMA 2001;285:2743-9.

10. Wraith JE, Tylki-Szymanska A, Guffon N, et al. Safety and efficacy of enzyme replacement therapy with agalsidase beta: an international, open-label study in pediatric patients with Fabry disease. J Pediatr 2008;152:563-70, 570.e1.

11. Eng CM, Banikazemi M, Gordon RE, et al. A phase $1 / 2$ clinical trial of enzyme replacement in fabry disease: pharmacokinetic, substrate clearance, and safety studies. Am J Hum Genet 2001;68:711-22.

12. Schiffmann R, Ries M, Timmons M, et al. Long-term therapy with agalsidase alfa for Fabry disease: safety and effects on renal function in a home infusion setting. Nephrol Dial Transplant 2006;21:345-54.

13. Schiffmann R, Hauer P, Freeman B, et al. Enzyme replacement therapy and intraepidermal innervation density in Fabry disease. Muscle Nerve 2006;34:53-6.

14. Feriozzi S, Schwarting A, Sunder-Plassmann G, et al. Agalsidase alfa slows the decline in renal function in patients with Fabry disease. Am J Nephrol 2009;29:353-61.

15. Feriozzi S, Torras J, Cybulla M, et al. The effectiveness of long-term agalsidase alfa therapy in the treatment of Fabry nephropathy. Clin J Am Soc Nephrol 2012;7:60-9.

16. Hughes DA, Barba Romero MA, Hollak CE, et al. Response of women with Fabry disease to enzyme replacement therapy: comparison with men, using data from FOS--the Fabry Outcome Survey. Mol Genet Metab 2011;103:207-14.

17. Smid BE, Rombach SM, Aerts JM, et al. Consequences of a global enzyme shortage of agalsidase beta in adult Dutch Fabry patients. Orphanet J Rare Dis 2011;6:69.

18. Eto Y, Ohashi T, Utsunomiya Y, et al. Enzyme replacement therapy in Japanese Fabry disease patients: the results of a phase 2 bridging study. J Inherit Metab Dis 2005;28:575-83.

19. Kampmann C, Perrin A, Beck M. Effectiveness of agalsidase alfa enzyme replacement in Fabry disease: cardiac outcomes after 10 years' treatment. Orphanet J Rare Dis 2015;10:125.

20. Beck M, Hughes D, Kampmann C, et al. Long-term effectiveness of agalsidase alfa enzyme replacement in Fabry disease: A Fabry Outcome Survey analysis. Mol Genet Metab Rep 2015;3:21-7.

21. Germain DP, Charrow J, Desnick RJ, et al. Ten-year outcome of enzyme replacement therapy with agalsidase beta in patients with Fabry disease. J Med Genet 2015;52:353-8.

22. Arends M, Biegstraaten M, Hughes DA, et al.
Retrospective study of long-term outcomes of enzyme replacement therapy in Fabry disease: Analysis of prognostic factors. PLoS One 2017;12:e0182379.

23. Arends M, Wijburg FA, Wanner C, et al. Favourable effect of early versus late start of enzyme replacement therapy on plasma globotriaosylsphingosine levels in men with classical Fabry disease. Mol Genet Metab 2017;121:157-61.

24. Weidemann F, Niemann M, Breunig F, et al. Longterm effects of enzyme replacement therapy on fabry cardiomyopathy: evidence for a better outcome with early treatment. Circulation 2009;119:524-9.

25. Niemann M, Rolfs A, Stork S, et al. Gene mutations versus clinically relevant phenotypes: lyso-Gb3 defines Fabry disease. Circ Cardiovasc Genet 2014;7:8-16.

26. Nowak A, Mechtler TP, Desnick RJ, et al. Plasma LysoGb3: A useful biomarker for the diagnosis and treatment of Fabry disease heterozygotes. Mol Genet Metab 2017;120:57-61.

27. Rombach SM, Aerts JM, Poorthuis BJ, et al. Long-term effect of antibodies against infused alpha-galactosidase $\mathrm{A}$ in Fabry disease on plasma and urinary (lyso)Gb3 reduction and treatment outcome. PLoS One 2012;7:e47805.

28. van Breemen MJ, Rombach SM, Dekker N, et al. Reduction of elevated plasma globotriaosylsphingosine in patients with classic Fabry disease following enzyme replacement therapy. Biochim Biophys Acta 2011;1812:70-6.

29. Goker-Alpan O, Nedd K, Shankar SP, et al. Effect and Tolerability of Agalsidase Alfa in Patients with Fabry Disease Who Were Treatment Naive or Formerly Treated with Agalsidase Beta or Agalsidase Alfa. JIMD Rep 2015;23:7-15.

30. Goker-Alpan O, Gambello MJ, Maegawa GH, et al. Reduction of Plasma Globotriaosylsphingosine Levels After Switching from Agalsidase Alfa to Agalsidase Beta as Enzyme Replacement Therapy for Fabry Disease. JIMD Rep 2016;25:95-106.

31. Kim JH, Lee BH, Hyang Cho J, et al. Long-term enzyme replacement therapy for Fabry disease: efficacy and unmet needs in cardiac and renal outcomes. J Hum Genet 2016;61:923-9.

32. Wilcox WR, Banikazemi M, Guffon N, et al. Long-term safety and efficacy of enzyme replacement therapy for Fabry disease. Am J Hum Genet 2004;75:65-74.

33. Choi JH, Cho YM, Suh KS, et al. Short-term efficacy of enzyme replacement therapy in Korean patients with Fabry disease. J Korean Med Sci 2008;23:243-50.

34. Kalliokoski RJ, Kantola I, Kalliokoski KK, et al. The effect of 12-month enzyme replacement therapy on myocardial 
perfusion in patients with Fabry disease. J Inherit Metab Dis 2006;29:112-8.

35. Vedder AC, Breunig F, Donker-Koopman WE, et al. Treatment of Fabry disease with different dosing regimens of agalsidase: effects on antibody formation and GL-3. Mol Genet Metab 2008;94:319-25.

36. Togawa T, Kodama T, Suzuki T, et al. Plasma globotriaosylsphingosine as a biomarker of Fabry disease. Mol Genet Metab 2010;100:257-61.

37. Schiffmann R, Swift C, Wang X, et al. A prospective 10-year study of individualized, intensified enzyme replacement therapy in advanced Fabry disease. J Inherit Metab Dis 2015;38:1129-36.

38. Schiffmann R, Askari H, Timmons M, et al. Weekly enzyme replacement therapy may slow decline of renal function in patients with Fabry disease who are on long-term biweekly dosing. J Am Soc Nephrol 2007;18:1576-83.

39. Linthorst GE, Germain DP, Hollak CE, et al. Expert opinion on temporary treatment recommendations for Fabry disease during the shortage of enzyme replacement therapy (ERT). Mol Genet Metab 2011;102:99-102.

40. Weidemann F, Kramer J, Duning T, et al. Patients with Fabry disease after enzyme replacement therapy dose reduction versus treatment switch. J Am Soc Nephrol 2014;25:837-49.

41. Pisani A, Spinelli L, Visciano B, et al. Effects of switching from agalsidase Beta to agalsidase alfa in 10 patients with anderson-fabry disease. JIMD Rep 2013;9:41-8.

42. Tsuboi K, Yamamoto H. Clinical observation of patients with Fabry disease after switching from agalsidase beta (Fabrazyme) to agalsidase alfa (Replagal). Genet Med 2012;14:779-86.

43. Krämer J, Lenders M, Canaan-Kuhl S, et al. Fabry disease under enzyme replacement therapy-new insights in efficacy of different dosages. Nephrol Dial Transplant 2018;33:1362-72.

44. Lenders M, Canaan-Kuhl S, Kramer J, et al. Patients with Fabry Disease after Enzyme Replacement Therapy Dose Reduction and Switch-2-Year Follow-Up. J Am Soc Nephrol 2016;27:952-62.

45. Lin HY, Huang YH, Liao HC, et al. Clinical observations on enzyme replacement therapy in patients with Fabry disease and the switch from agalsidase beta to agalsidase alfa. J Chin Med Assoc 2014;77:190-7.

46. Tsuboi K, Yamamoto H. Clinical course of patients with Fabry disease who were switched from agalsidase-beta to agalsidase-alpha. Genet Med 2014;16:766-72.

47. Pisani A, Bruzzese D, Sabbatini M, et al. Switch to agalsidase alfa after shortage of agalsidase beta in Fabry disease: a systematic review and meta-analysis of the literature. Genet Med 2017;19:275-82.

48. Lenders M, Oder D, Nowak A, et al. Impact of immunosuppressive therapy on therapy-neutralizing antibodies in transplanted patients with Fabry disease. J Intern Med 2017;282:241-53.

49. Mauhin W, Lidove O, Masat E, et al. Innate and Adaptive Immune Response in Fabry Disease. JIMD Rep 2015;22:1-10.

50. Bénichou B, Goyal S, Sung C, et al. A retrospective analysis of the potential impact of $\operatorname{IgG}$ antibodies to agalsidase beta on efficacy during enzyme replacement therapy for Fabry disease. Mol Genet Metab 2009;96:4-12.

51. Linthorst GE, Hollak CE, Donker-Koopman WE, et al. Enzyme therapy for Fabry disease: neutralizing antibodies toward agalsidase alpha and beta. Kidney Int 2004;66:1589-95.

52. Linthorst GE, Aerts JM. Letter concerning "Enzyme replacement therapy in a patient with Fabry disease and the development of $\mathrm{IgE}$ antibodies against agalsidase beta but not agalsidase alpha", by Tanaka et al. J Inherit Metab Dis 2011;34:237-8.

53. Tanaka A, Takeda T, Hoshina T, et al. Enzyme replacement therapy in a patient with Fabry disease and the development of $\mathrm{IgE}$ antibodies against agalsidase beta but not agalsidase alpha. J Inherit Metab Dis 2010;33 Suppl 3:S249-52.

54. Tesmoingt C, Lidove O, Reberga A, et al. Enzyme therapy in Fabry disease: severe adverse events associated with anti-agalsidase cross-reactive IgG antibodies. Br J Clin Pharmacol 2009;68:765-9.

55. Germain DP, Fan JQ. Pharmacological chaperone therapy by active-site-specific chaperones in Fabry disease: in vitro and preclinical studies. Int J Clin Pharmacol Ther 2009;47 Suppl 1:S111-7.

56. Benjamin ER, Della Valle MC, Wu X, et al. The validation of pharmacogenetics for the identification of Fabry patients to be treated with migalastat. Genet Med 2017;19:430-8.

57. Germain DP, Hughes DA, Nicholls K, et al. Treatment of Fabry's Disease with the Pharmacologic Chaperone Migalastat. N Engl J Med 2016;375:545-55.

58. Hughes DA, Nicholls K, Shankar SP, et al. Oral pharmacological chaperone migalastat compared with enzyme replacement therapy in Fabry disease: 18-month results from the randomised phase III ATTRACT study. J Med Genet 2017;54:288-96. 
59. Germain DP, Nicholls K, Giugliani R, et al. Efficacy of the pharmacologic chaperone migalastat in a subset of male patients with the classic phenotype of Fabry disease and migalastat-amenable variants: data from the phase 3 randomized, multicenter, double-blind clinical trial and extension study. Genet Med 2019;21:1987-97.

60. Müntze J, Salinger T, Gensler D, et al. Treatment of hypertrophic cardiomyopathy caused by cardiospecific variants of Fabry disease with chaperone therapy. Eur Heart J 2018;39:1861-2.

61. Müntze J, Gensler D, Maniuc O, et al. Oral Chaperone Therapy Migalastat for Treating Fabry Disease: Enzymatic Response and Serum Biomarker Changes After 1 Year. Clin Pharmacol Ther 2019;105:1224-33.

62. Müntze J, Nordbeck P. Response to "Oral Chaperone Therapy Migalastat for the Treatment of Fabry Disease: Potentials and Pitfalls of Real-World Data". Clin Pharmacol Ther 2019;106:927-8.

63. Lenders M, Nordbeck P, Kurschat C, et al. Treatment of Fabry disease with migalastat - outcome from a prospective observational multicenter study (FAMOUS). Clin Pharmacol Ther 2020;108:326-37.

64. Jain G, Warnock DG. Blood pressure, proteinuria and nephropathy in Fabry disease. Nephron Clin Pract 2011;118:c43-8.

65. Warnock DG, Thomas CP, Vujkovac B, et al. Antiproteinuric therapy and Fabry nephropathy: factors associated with preserved kidney function during agalsidase-beta therapy. J Med Genet 2015;52:860-6.

66. Wanner C, Breunig F. Fabry nephropathy and the case for adjunctive renal therapy. J Am Soc Nephrol 2007;18:2426-8.

67. Ersözlü S, Desnick RJ, Huynh-Do U, et al. Long-term Outcomes of Kidney Transplantation in Fabry Disease. Transplantation 2018;102:1924-33.

68. Schiffmann R, Warnock DG, Banikazemi M, et al. Fabry disease: progression of nephropathy, and prevalence of cardiac and cerebrovascular events before enzyme replacement therapy. Nephrol Dial Transplant 2009;24:2102-11.

69. Ponikowski P, Voors AA, Anker SD, et al. 2016 ESC Guidelines for the diagnosis and treatment of acute and chronic heart failure: The Task Force for the diagnosis and treatment of acute and chronic heart failure of the European Society of Cardiology (ESC)Developed with the special contribution of the Heart Failure Association (HFA) of the ESC. Eur Heart J 2016;37:2129-200.

70. De Angelis G, Tsoumani Z, Reid A, et al. Late-onset Fabry disease presenting with ventricular tachycardia originating from typical inferolateral scar. Can J Cardiol 2020;36:1832. e1-1832.e4.

71. Vijapurapu R, Geberhiwot T, Jovanovic A, et al. Study of indications for cardiac device implantation and utilisation in Fabry cardiomyopathy. Heart 2019;105:1825-31.

72. Pavlu L, Kocourkova L, Taborsky M, et al. Ventricular tachycardia: a presentation of Fabry disease case report. Eur Heart J Case Rep 2018;3:yty154.

73. Silva-Gburek J, Rochford L, Hopkin R, Jefferies JL. Ventricular Tachycardia in Fabry Disease Detected in a 50-Year-Old Woman during 14-Day Continuous Cardiac Monitoring. Tex Heart Inst J 2016;43:531-3.

74. Oder D, Liu D, Hu K, et al. alpha-Galactosidase A Genotype N215S Induces a Specific Cardiac Variant of Fabry Disease. Circ Cardiovasc Genet 2017;10:e001691.

75. Rolfs A, Bottcher T, Zschiesche M, et al. Prevalence of Fabry disease in patients with cryptogenic stroke: a prospective study. Lancet 2005;366:1794-6.

76. Powers WJ, Rabinstein AA, Ackerson T, et al. Guidelines for the Early Management of Patients With Acute Ischemic Stroke: 2019 Update to the 2018 Guidelines for the Early Management of Acute Ischemic Stroke: A Guideline for Healthcare Professionals From the American Heart Association/American Stroke Association. Stroke 2019;50:e344-e418.

77. Üçeyler N, Ganendiran S, Kramer D, et al. Characterization of pain in fabry disease. Clin J Pain 2014;30:915-20.

78. Shen JS, Busch A, Day TS, et al. Mannose receptormediated delivery of moss-made alpha-galactosidase A efficiently corrects enzyme deficiency in Fabry mice. J Inherit Metab Dis 2016;39:293-303.

79. Shaaltiel Y, Bartfeld D, Hashmueli S, et al. Production of glucocerebrosidase with terminal mannose glycans for enzyme replacement therapy of Gaucher's disease using a plant cell system. Plant Biotechnol J 2007;5:579-90.

80. Du H, Cameron TL, Garger SJ, et al. Wolman disease/ cholesteryl ester storage disease: efficacy of plantproduced human lysosomal acid lipase in mice. J Lipid Res 2008;49:1646-57.

81. He X, Haselhorst T, von Itzstein M, et al. Production of alpha-L-iduronidase in maize for the potential treatment of a human lysosomal storage disease. Nat Commun 2012;3:1062.

82. Schiffmann R, Goker-Alpan O, Holida M, et al. Pegunigalsidase alfa, a novel PEGylated enzyme replacement therapy for Fabry disease, provides sustained 
plasma concentrations and favorable pharmacodynamics: A 1-year Phase 1/2 clinical trial. J Inherit Metab Dis 2019;42:534-44.

83. Kizhner T, Azulay Y, Hainrichson M, et al. Characterization of a chemically modified plant cell culture expressed human alpha-Galactosidase-A enzyme for treatment of Fabry disease. Mol Genet Metab 2015;114:259-67.

84. Linhart A, Dostalova G, Nicholls K, et al. Switching from agalsidase alfa to pegunigalsidase alfa for treating Fabry disease - one year of treatment: data from BRIDGE - a phase III open label study. Mol Genet Metab 2020;129:S98-S99.

85. Reski R, Parsons J, Decker EL. Moss-made pharmaceuticals: from bench to bedside. Plant Biotechnol J 2015;13:1191-8.

86. Hennermann JB, Arash-Kaps L, Fekete G, et al. Pharmacokinetics, pharmacodynamics, and safety of mossaGalactosidase A in patients with Fabry disease. J Inherit Metab Dis 2019;42:527-33.

87. Cox TM. Innovative treatments for lysosomal diseases. Best Pract Res Clin Endocrinol Metab 2015;29:275-311.

88. Platt FM, Jeyakumar M, Andersson U et al. Inhibition of substrate synthesis as a strategy for glycolipid lysosomal storage disease therapy. J Inherit Metab Dis 2001;24:275-90.

89. Ashe KM, Budman E, Bangari DS, et al. Efficacy of Enzyme and Substrate Reduction Therapy with a Novel Antagonist of Glucosylceramide Synthase for Fabry Disease. Mol Med 2015;21:389-99.

90. Marshall J, Ashe KM, Bangari D, et al. Substrate reduction augments the efficacy of enzyme therapy in a mouse model of Fabry disease. PLoS One 2010;5:e15033.

91. Guérard N, Morand O, Dingemanse J. Lucerastat, an iminosugar with potential as substrate reduction therapy for glycolipid storage disorders: safety, tolerability, and pharmacokinetics in healthy subjects. Orphanet J Rare Dis
2017;12:9.

92. Deegan P GD, Goker-Alpan O, et al. Three year open label phase 2 a investigation of venglustat safety and exploratory efficacy in classic Fabry patients. Book of abstracts SSIEM 2019, JIMD 42 2019; Suppl1 9:O-019.

93. Guérard N, Oder D, Nordbeck P, et al. Lucerastat, an Iminosugar for Substrate Reduction Therapy: Tolerability, Pharmacodynamics, and Pharmacokinetics in Patients With Fabry Disease on Enzyme Replacement. Clin Pharmacol Ther 2018;103:703-11.

94. Ruiz de Garibay AP, Solinis MA, Rodriguez-Gascon A. Gene therapy for fabry disease: a review of the literature. BioDrugs 2013;27:237-46.

95. Huston MW, Yasuda M, Pagant S, et al. Liver-targeted AAV gene therapy vectors produced by a clinical scale manufacturing process result in high, continuous therapeutic levels of enzyme activity and effective substrate reduction in mouse model of Fabry disease. Mol Genet Metab 2019;2:S77.

96. Kia A, McIntosh J, Rosales C, et al. Efficacy evaluation of liver-directed gene therapy in Fabry mice. Blood 2018;132:2209.

97. van der Veen SJ, Hollak CEM, van Kuilenburg ABP, Langeveld M. Developments in the treatment of Fabry disease. J Inherit Metab Dis 2020;43:908-21.

98. Nabhan JF, Wood KM, Rao VP, et al. Intrathecal delivery of frataxin mRNA encapsulated in lipid nanoparticles to dorsal root ganglia as a potential therapeutic for Friedreich's ataxia. Sci Rep 2016;6:20019.

99. Zhu X, Yin L, Theisen M, et al. Systemic mRNA Therapy for the Treatment of Fabry Disease: Preclinical Studies in Wild-Type Mice, Fabry Mouse Model, and Wild-Type Non-human Primates. Am J Hum Genet 2019;104:625-37.

100.DeRosa F, Smith L, Shen Y, et al. Improved Efficacy in a Fabry Disease Model Using a Systemic mRNA Liver Depot System as Compared to Enzyme Replacement Therapy. Mol Ther 2019;27:878-89.
Cite this article as: Oder D, Müntze J, Nordbeck P. Contemporary therapeutics and new drug developments for treatment of Fabry disease: a narrative review. Cardiovasc Diagn Ther 2021;11(2):683-695. doi: 10.21037/cdt-20-743 